\title{
Fatores determinantes do absenteísmo de pacientes às consultas agendadas na Unidade Básica de Saúde Laranjeiras, Marabá, Pará
}

\author{
Determining factors of patient absenteeism at appointments scheduled at the Laranjeiras Basic \\ Health Unit, Marabá, Pará
}

Factores determinantes de absentismo de pacientes em citas programadas em la Unidad Básica de

Salud Laranjeiras, Marabá, Pará

Recebido: 29/05/2021 | Revisado: 06/06/2021 | Aceito: 08/06/2021 | Publicado: 22/06/2021

\author{
Isaac Antonio Duarte da Silva \\ ORCID: https://orcid.org/0000-0002-1123-043X \\ Universidade do Estado do Pará, Brasil \\ E-mail: isaacduarte480@gmail.com \\ Pedro Rafael Rocha Stermer \\ ORCID: https://orcid.org/0000-0003-0595-0585 \\ Universidade do Estado do Pará, Brasil \\ E-mail: pedrostermer123@gmail.com \\ Larissa Navarro Barros \\ ORCID: https://orcid.org/0000-0003-1646-8997 \\ Universidade do Estado do Pará, Brasil \\ E-mail: larissa.navarro@uepa.br \\ Sarah Lais Rocha \\ ORCID: https://orcid.org/0000-0002-3753-9507 \\ Universidade do Estado do Pará, Brasil \\ Faculdade Carajás, Brasil \\ E-mail: sarahlaisrocha@gmail.com \\ Robson José de Souza Domingues \\ ORCID: https://orcid.org/0000-0001-5419-2878 \\ Universidade do Estado do Pará, Brasil \\ E-mail: domingues@uepa.br
}

\begin{abstract}
Resumo
O absenteísmo do usuário no sistema de saúde consiste no ato de não comparecer às consultas e aos procedimentos agendados no SUS. O objetivo do projeto foi identificar os fatores que influenciam no absenteísmo de pacientes de UBS em consultas marcadas. Trata-se de um estudo exploratório, descritivo e de abordagem qualitativa. O instrumento de coleta foi a entrevista por telefone, estes foram retirados dos cadernos de consulta da UBS Laranjeiras - Marabá/PA. Foi feita análise estatística descritiva do perfil dos participantes e análise de conteúdo para pesquisar os motivos das faltas e o entendimento do paciente acerca das consequências deste ato. O perfil dos participantes foi principalmente feminino (75\%), não branco (96,8\%), com uma média de idade de 33,9 anos e 66,7\% eram desempregados no momento da entrevista. Foram encontrados como motivadores do absenteísmo a gestão da unidade, a inadequação dos horários e datas marcados, a dificuldade com o deslocamento dos usuários à UBS, fatores ambientais, a relação médico-paciente e o desconhecimento do paciente acerca dos procedimentos. A percepção dos pacientes sobre as consequências de seu absenteísmo mostrou usuários que reconheceram o dano que poderia ser causado a outros usuários ou a si mesmo e pacientes que desconheciam ou negligenciavam estes danos. For fim, devese reconhecer que há dificuldades em relação a comunicação entre o usuário e a UBS e, assim, procurar alternativas que diminuam ruídos existentes e que possibilitem um melhor contato entre os sujeitos.
\end{abstract}

Palavras-chave: Absenteísmo; Saúde básica; Paciente.

\begin{abstract}
User absenteeism in the health system consists of not attending the scheduled appointments and scheduled procedures at SUS. The objective of this project was to identify the factors that influence the absenteeism of UBS patients in scheduled appointments. This is an exploratory, descriptive study with a qualitative approach. The collection instrument was telephone interview, which one was taken from the consultation books of UBS Laranjeiras - Marabá / PA. Descriptive statistical analysis of the participants' profile and content analysis were used to research the reasons for absences and the patient's understanding of this act consequences. The profile of the participants was mainly female $(75 \%)$, non-white $(96.8 \%)$, with a mean age of 33.9 years and $66.7 \%$ were unemployed at the time of the interview. The management of the unit, the inadequacy of the scheduled appointments dates, the difficulty with the
\end{abstract}


users' displacement to the UBS, environmental factors, the doctor-patient relationship and the patient's lack of knowledge about the procedures were found as motivators of absenteeism. Patients' perception of absenteeism consequences showed users who recognized the damage that could be caused to other users or themselves and patients who were unaware of or neglected this damage. Finally, it must be recognized that there are difficulties in relation to the communication between the user and the UBS and, thus, look for alternatives that reduce existing noise and that allow a better contact between the subjects.

Keywords: Absenteeism; Public health; Patient.

\section{Resumen}

El absentismo de los usuarios en el sistema de salud consiste en no asistir a las citas y trámites programados en el SUS. El objetivo del proyecto fue identificar los factores que influyen en el absentismo de los pacientes con SBU en las citas programadas. Se trata de un estudio exploratorio, descriptivo con enfoque cualitativo. El instrumento de recaudación fue la entrevista telefónica, estas fueron tomadas de los libros de consulta de la UBS Laranjeiras - Marabá / PA. Se realizó un análisis estadístico descriptivo del perfil de los participantes y un análisis de contenido para investigar los motivos de las ausencias y la comprensión del paciente sobre las consecuencias de este acto. El perfil de los participantes fue mayoritariamente femenino $(75 \%)$, no blanco $(96,8 \%)$, con una edad media de 33,9 años y el $66,7 \%$ estaban desempleados en el momento de la entrevista. La gestión de la unidad, la inadecuación de los horarios y fechas programadas, la dificultad con el desplazamiento de los usuarios a la UBS, los factores ambientales, la relación médico-paciente y el desconocimiento del paciente sobre los procedimientos se encontraron como motivadores del absentismo. La percepción de los pacientes sobre las consecuencias de su absentismo mostró a los usuarios que reconocían el daño que podía ocasionar a otros usuarios oa ellos mismos y a los pacientes que desconocían o desatendían este daño. Finalmente, hay que reconocer que existen dificultades en relación a la comunicación entre el usuario y la UBS y, así, buscar alternativas que reduzcan el ruido existente y que permitan un mejor contacto entre los sujetos.

Palabras clave: Absentismo; Salud básica; Paciente.

\section{Introdução}

O absenteísmo do usuário no sistema de saúde consiste no ato de não comparecer às consultas e aos procedimentos agendados no SUS. Esta prática gera desperdícios de recursos públicos, desorganiza a oferta de serviços, limita a garantia da atenção nos diversos níveis de assistência e retorno dos usuários faltosos ao fluxo de marcações de consultas e exames (Costa et al., 2018).

O índice de não comparecimento às consultas de saúde está relacionado a diversos fatores e varia dependendo da localidade, da especialidade médica e da faixa etária do paciente. As informações disponíveis indicam que esse é um problema crônico e que se estende por todo o país, sendo esses fatores de contribuição para a relevância deste estudo (Costa, 2018)

Anteriormente a criação do SUS, o Ministério da Saúde utilizava, juntamente com os apoios municipais e estaduais ações de promoção de saúde com o enfoque em campanhas de vacinação e combate a endemias nas quais diversas vezes o alvo era o vetor da doença (Brasil, 2017).

A Estratégia de Saúde da Família (ESF) é um dos programas governamentais que foram propostos com o intuito de promover maior alcance da saúde à população. Essa estratégia objetiva aproximar a população do atendimento e, para isso, o projeto contém uma equipe multiprofissional que fica responsável por uma parcela especifica de moradores de uma cidade, em uma região delimitada (Brasil, 2017).

As equipes de saúde da família podem atuar tanto nas UBS quanto em locais da comunidade, como a própria residência dos adscritos. Esta proximidade entre profissionais e população permite a identificação mais precisa das reais necessidades da localidade e possibilita planejamento mais adequado das formas de abordagem, podendo trabalhar com a prevenção e a promoção da saúde, além de suprir boa parte da demanda por atendimento (Macinko \& Mendonça, 2018).

As unidades devem estar preparadas para o atendimento tanto de demanda espontânea, bem como das consultas agendadas. Essa dinâmica permite, novamente, que a UBS seja a forma de contato preferencial para quem procura atendimento público. Desta forma, o agendamento das consultas deve adaptar-se a demanda por atendimento. As consultas são realizadas de 
forma multidisciplinar e podem ser direcionadas a médicos e enfermeiros, sendo uma das atribuições desses, além de algum outro profissional vinculado a unidade (Brasil, 2017).

As consultas podem ser agendadas tanto por um agente comunitário de saúde quanto pela recepção da UBS, esta última modalidade exige o deslocamento do cidadão para a unidade e o conhecimento do mesmo acerca dinâmica de dias de agendamento de consultas, pois a instituição tem flexibilidade para organizar os serviços oferecidos de modo a adaptar-se as principais necessidades da região de abrangência. Por esta maleabilidade, é esperado que cidadãos desinformados não consigam agendar suas consultas ou utilizar de um serviço da forma como esperava. Por essa razão, é importante a sinalização destas informações por meio de cartazes e afins anexados em murais ou outros locais de fácil visualização nas áreas de maior circulação da UBS (Brasil, 2017).

O presente estudo justifica-se através de uma problemática analisada durante as atividades do módulo de Gestão, Interação Ensino, Serviço e Comunidade (GIESC), uma disciplina da matriz curricular do curso de graduação em Medicinada da Universidade do Estado do Pará (UEPA), onde o acadêmico tem a oportunidade de interagir desde o início da sua formação com a comunidade de forma mais concreta, acompanhando todas as facetas da ESF nas UBS. A vivência dessa disciplina possibilitou realizar observações pontuais sobre a importância do paciente comparecer as consultas agendadas, pois houve, durante esse período no Centro de Saúde Laranjeiras, a percepção empírica de um elevado número de não comparecimento de usuários às consultas e exames agendados, o que compromete o trabalho da equipe de saúde e prejudica outros cidadãos que não conseguiram agendar $\mathrm{o}$ atendimento.

Desse modo, é prejudicial a não efetivação da presença e compromisso dos pacientes nas consultas agendadas com uma equipe multiprofissional, haja vista a alta demanda na região. A presença do paciente nas consultas agendadas e o seu comprometimento colabora para a dinâmica funcional adequada das unidades, que tem como pressuposto a validação das qualidades profissionais dos componentes das equipes de saúde e a promoção de saúde da comunidade.

Além disso, os resultados podem identificar os fatores que contribuem para o absenteísmo dos pacientes na UBS e, a partir disso, direcionar para possíveis motivações do não comparecimento. Com essas informações, é possível sugerir ações e medidas que visem amenizar o fato e, assim, melhorar o funcionamento da UBS. A pesquisa também pode servir como incentivo para que outros estudos similares sejam realizados e que se esclareça, cada vez mais, os fatores determinantes que corroboram para a falta de pacientes às consultas agendadas.

Vale, também, ressaltar a importância curricular e formativa deste trabalho, pois é um pré-requisito para a conclusão do ano letivo do curso. Além disso, essa pesquisa contribui de forma exponencial para a formação curricular dos seus idealizadores

Baseando-se no que foi exposto e levando em consideração a seguinte pergunta problema: Quais são os motivos que levam pacientes a faltarem a consultas pré-agendadas na Unidade Básica de Saúde? O objetivo geral deste estudo foi identificar os fatores que influenciam no absenteísmo de pacientes de UBS em consultas marcadas, e os objetivos específicos foram: identificar o perfil dos pacientes absentes de consultas ambulatoriais na Unidade Básica de Saúde e entender as motivações do absenteísmo dos pacientes pautando as condições relativas aos seus perfis.

\section{Metodologia}

Trata-se de um estudo exploratório, descritivo, com abordagem qualitativa, tendo como foco os usuários do Sistema Único de Saúde do município de Marabá, absentes às consultas na Unidade Básica de Saúde Laranjeiras.

Um estudo exploratório trata-se de uma modalidade que procura gerar evidências que colaborem para a produção de pesquisas com maiores escalas, tomando caráter introdutório e sendo usado para analisar a viabilidade da mesma frente científica (Hallingberg et al., 2018). 
Um trabalho de descrição qualitativa tem como suas características uma metodologia flexível que procura examinar um fenômeno com um olhar naturalista. Estes podem ser complementados, em sua análise, com dados quantitativos, a fim de enriquecer a pesquisa (Kim et al., 2017).

\subsection{Amostra}

A amostra referente à coleta de dados através dos cadernos de consultas, que foi definida considerando o número total de faltosos registrados no período e calculando o número de casos necessários para amostragem. Outrossim, a seleção dos indivíduos que responderam a entrevista foi realizada de forma aleatória, considerando critérios de inclusão e exclusão da pesquisa. O cálculo da amostragem foi feito utilizando o cálculo para populações finitas (Figura 1). Foi adotado a percentagem do fenômeno padrão (50\%), erro máximo de 5\% e nível de confiança de $95 \%$.

Figura 1 - Cálculo do tamanho da amostra para populações finitas.

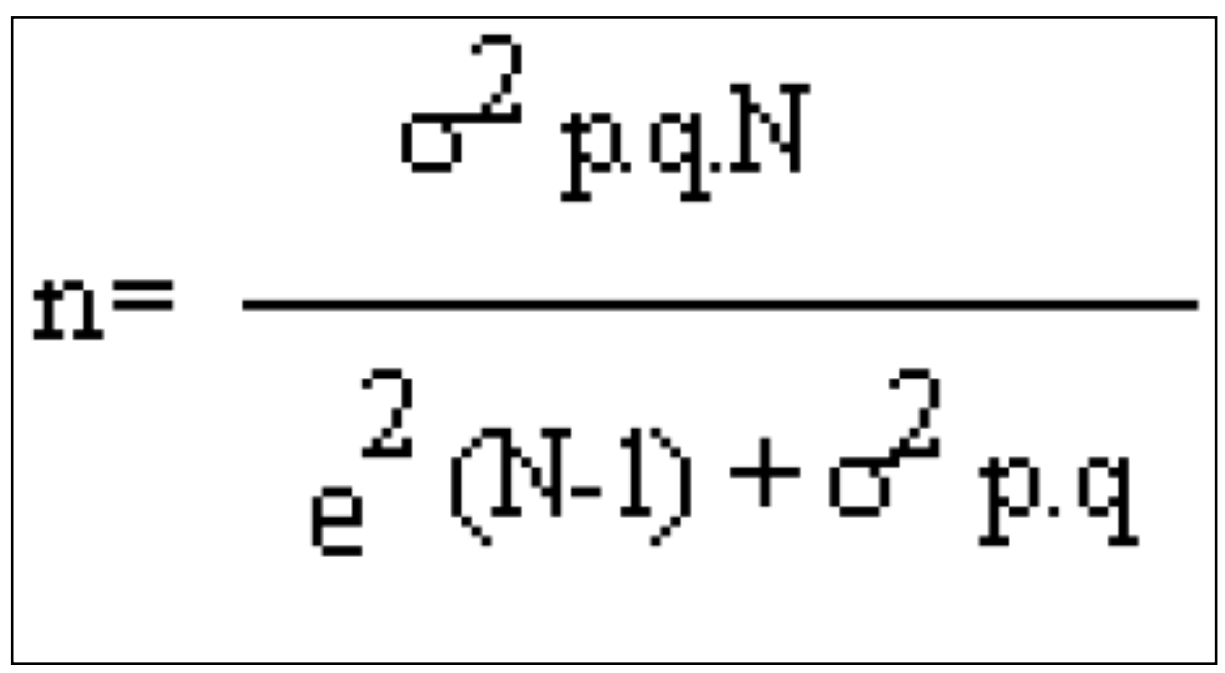

Legenda: $\mathrm{n}$ = tamanho da amostra; $\sigma$ = nível de confiança escolhido, expresso em números de desvios padrão; $\mathrm{p}=$ percentagem do fenómeno; $\mathrm{q}=$ percentagem complementar; $\mathrm{N}=$ tamanho da população; e = erro máximo permitido. Fonte: (Pocinho \& Figueredo, 2004).

As entrevistas foram realizadas, em ordem aleatória, buscando alcançar o tamanho amostral calculado ou o máximo de entrevistas completas, caso este número de entrevistas bem sucedidas não chegue à amostra ideal.

A relação de usuários faltosos no período foi obtida através do levantamento dos dados das agendas de consultas, no período predeterminado. Se necessário, os pesquisadores recorreriam aos prontuários dos pacientes para terem acesso aos números de telefone.

\subsection{Período}

A coleta de dados ocorreu no mês de março de 2020 mediante aprovação do Comitê de Ética em Pesquisa. Entretanto, pelo fato de que os documentos analisados terem caráter de registro, os cadernos que foram analisados continham informações que cobriam o período de dezembro de 2019 a fevereiro de 2020.

\subsection{Critérios de inclusão e exclusão}

Os critérios de inclusão são: (a) pacientes que faltaram a uma consulta registrada nos cadernos de agendamentos durante o período predeterminado; (b) pacientes que aceitarem participar da pesquisa e terem registrado o aceite do Termo de Consentimento Livre e Esclarecido (ANEXO - D). 
A verificação de casos viáveis para estudo foi realizada eliminando: (a) usuários repetidos; (b) usuários sem endereço ou com duplo endereço; (c) usuários sem telefone; (d) usuários menores de 18 anos; (e) usuários que morreram; (f) usuários com deficiência mental; (g) usuários que não recordem terem faltado a consulta referida na entrevista.

\subsection{Aspectos éticos}

Esta pesquisa atende os princípios éticos e respeitará os preceitos da Declaração de Helsinque (1964) e Código de Nuremberg (1947), considerando todas as normas de pesquisa envolvendo seres humanos (Resolução CNS466/12) do Conselho Nacional de Saúde (BRASIL, 2012). Isso, prontamente, após o aceita da orientadora do projeto. Aos indivíduos entrevistados, houve a aplicação do termo de consentimento livre e esclarecido. Há o comprometimento com o mínimo de dano e o máximo de benefício possível e a garantia de se evitar danos previsíveis. Deste modo, a pesquisa só foi desenvolvida após a aprovação pelo Conselho de Ética em Pesquisa (CEP). CAAE: 12937919.6.0000.8607

\subsection{Local de estudo}

O local de estudo foi o Centro de Saúde Laranjeiras. Localizado na rua José Bandeira de Souza, 2-128 - Bairro Laranjeiras, Marabá-PA. Marabá é um município brasileiro localizado no sudeste do estado do Pará, região norte do país. É o município sede da região metropolitana de Marabá. Se localiza cerca de 500 quilômetros ao sul da capital do estado. Sua localização tem, por referência, o ponto de encontro entre dois grandes rios, Tocantins e Itacaiúnas, formando uma espécie de "Y" no seio da cidade vista de cima. É formada basicamente por seis distritos urbanos interligados por rodovias. É o quarto mais populoso do Pará, com 275.086 habitantes, segundo o Instituto Brasileiro de Geografia e Estatística (IBGE, 2010), e com o $3^{\circ}$ maior produto interno bruto (PIB) do estado em 2015, com 7,3 bilhões de reais. O seu Índice de Desenvolvimento Humano (IDH) é 0,668, considerado médio pelo Programa das Nações Unidas para o Desenvolvimento (PNUD) (IBGE, 2010). Sua renda per capita em 2015 era de 27.956,09 reais. É o principal centro socioeconômico do sudeste paraense e um dos municípios mais dinâmicos do Brasil. A seguir, mais informações sobre a unidade:

Centro de Saúde Laranjeiras é uma unidade de atendimento à saúde do tipo Centro de Saúde, Unidade Básica, estando cadastrado no Ministério da Saúde sob o número 2614774 e que está apta a prestar serviços de Controle de Tabagismo, Pré-natal/Parto e Nascimento, Agentes Comunitários de Saúde, Tratamento da Tuberculose à população na região do bairro Laranjeiras da cidade Marabá - PA (UBS.MED.BR, 2018, p. 1).

Citando as características da cidade de Marabá, cabe ressaltar as análises realizadas pelo autor regional Airton Pereira dos Reis (2015) que, diante de andanças e observações realizadas pelas diversas áreas do município conseguiu traçar um perfil e identificar as principais características que regem a cidade. O autor, descreve Marabá como uma cidade formada por bairros heterogêneos, de casas precárias, em sua maioria, sem água tratada com esgoto fluindo pelas ruas sem nenhum tipo de saneamento que contemple a grande massa marginalizada da população formada, via de regra, por camponeses, trabalhadores pobres e migrantes que assumiram o desejo de novas e melhores condições de vida na terra bendita.

A UBS foi escolhida devido ao maior contato dos pesquisadores com a localidade, visto que é espaço de realização do módulo GIESC, e também é ambiente de trabalho da professora/orientadora. Estes fatos permitem mais fácil acesso ao local e, consequentemente, aos abjetos de estudo.

\subsection{Riscos e Benefícios}

Há o risco de extravasamento de dados presentes nos documentos. Na entrevista também corre os riscos de exposição ou extravasamento do conteúdo gravado ou o vazamento do nome do entrevistado. Contudo, precações foram tomadas para 
que estes sejam minimizados, o manuseio dos documentos da UBS foi realizado no local, o conteúdo das gravações, bem como qualquer texto relativo a esta foi salvo em uma conta na nuvem, com senha.

Os benefícios são: demonstrar dados que podem ser úteis para o direcionamento mais adequado de políticas públicas que procurem amenizar o problema do absenteísmo dos pacientes, podendo também encontrar problemas específicos que podem ser tratados pela equipe da UBS; este trabalho também é uma contribuição para a academia, visto que traz dados que podem servir de auxílio para futuros trabalhos; por fim, também contribui para o conhecimento dos realizadores tanto a respeito do tema quanto a respeito da construção de uma pesquisa em si.

\subsection{Materiais e Métodos}

Primeiramente foi realizada uma análise documental a fim de identificar e selecionar possíveis paciente a serem entrevistados e, também, dimensionar o tamanho da população para a realização do cálculo amostral. Para coletar essas informações, foram utilizados os cadernos de consulta da UBS e, se necessário, os prontuários dos pacientes.

A análise documental consiste na utilização de registros documentados para extrair informações com um objetivo específico, podendo ou não ser de diferentes fontes e formatos, procurando refletir as informações das fontes originais (Cechinel et al., 2016).

A partir destes dados obtidos, foram selecionados, entres os pacientes absentes, um grupo amostral que foi aplicado a entrevista via telefone para que seja possível levantar tanto variáveis classificatórias quanto os principais motivos pelo o qual os pacientes foram ausentes nas consultas.

A entrevista torna possível acessar informações de forma direta, permitindo que o entrevistado exponha sua perspectiva sobre um assunto. Além disso, permite a captura as complexidades dos fenômenos e seu impacto no entrevistado (Troncoso-Pantoja \& Amaya-Placencia, 2017).

A entrevista por telefone apresenta várias vantagens. Dentre estas, destacam-se os custos reduzidos para realização do contato, a facilidade na seleção da amostra, a rapidez na execução, o que reflete numa economia de recursos financeiros na coleta de dados. Além disso, permite o alcance de mais pessoas que estariam impossibilitadas de serem contactadas por outra forma (Batista et al., 2017).

Antes que a coleta de dados ocorra, o entrevistado foi devidamente informado sobre o teor da pesquisa. Primeiramente foi feita uma pequena apresentação do pesquisador, informando seu nome e a instituição da pesquisa, no caso a UEPA, Campus Marabá. Seguindo, foi esclarecido rapidamente que se trata de uma pesquisa e que o entrevistado possui o direito de pedir para que seja interrompida sem qualquer punição. Também foi informado que a entrevista estava sendo gravada e que seus dados estão seguros, podendo também solicitar a exclusão da gravação. Então foi lido o Termo de Consentimento Livre e Esclarecido (ANEXO - D). Após essa etapa, foi questionado ao participante se ele gostaria de continuar, para tal, o entrevistador proferiu a seguinte frase: "Senhor(a) , declara que concorda em participar deste estudo?". Se a resposta fosse negativa, a entrevista acabava por ali e os dados seriam excluídos. Se a resposta fosse positiva, após a reafirmação do aceite em participar da pesquisa, a entrevista continuaria. O participante também foi informado de que ele poderia solicitar uma cópia tanto do termo quanto da gravação via e-mail ou rede social.

O instrumento de coleta de dados foi a entrevista semiestruturada. Esta consiste em uma modalidade que, apesar de contar com um roteiro prévio, é flexível e permite ao pesquisador guiar a conversa de forma a atingir seus objetivos. Ademais, possibilita um aprofundamento devido a interação mais orgânica entre pesquisador e pesquisado (Batista et al., 2017).

$\mathrm{Na}$ intenção de manter o sigilo de cada entrevistado, os participantes foram identificados como FALTOSO (FAL), seguidos por ordem numérica 01, 02, 03 e assim por diante (exemplo: FAL_01, FAL_02, FAL_03). A entrevista é composta de perguntas mais fechadas e abertas. A finalidade das perguntas fechadas é recolher dados sobre o perfil dos pacientes utilizando 
as seguintes variáveis: idade; sexo; cor; escolaridade; presença ou não de condição ou doença; posse de veículo; número de filhos; trabalho; responsável por dependente. Os dados referentes a classificação e as variáveis foram registrados no programa Excel® para a criação de um banco de dados para análise posterior.

A entrevista apresenta também questões abertas e semiestruturadas, construídas para nortear a problemática do absenteísmo dos pacientes. A escolha das perguntas teve como base o roteiro elaborado por Gonçalves et al. (2015) em sua pesquisa. O objetivo destas perguntas é procurar compreender os motivos pelos quais os pacientes faltaram as consultas e busca o entendimento do entrevistado acerca das consequências que seu absenteísmo pode causar na UBS.

As gravações foram realizadas com o intermédio da aplicação Gravador de Chamadas (Call Recorder). Após ser instalado, pela Play Store ou a Apple Store. O aplicativo foi habilitado a gravar todas as chamadas realizadas pelo telefone do momento em diante, notificando o usuário após as chamadas forem gravadas. A função de gravar automaticamente pode ser desabilitada. Desta forma, o aplicativo permanece com a função desabilitada e é reabilitada apenas no momento em que as entrevistas ocorrerem. Após o termino de cada ligação, as gravações são salvas e armazenadas temporariamente na memória dos celulares, e são enviadas para um drive de nuvem.

As entrevistas foram transcritas logo após a sua realização, ou no momento mais próximo possível depois de sua execução. Para tanto, foi utilizado o programa de produção de documentos digitais Word®. Neste, todas as entrevistas foram transcritas de forma que cada uma dessas esteja em um documento separado, que foi identificado da forma descrita anteriormente (ex: FAL_01, FAL_02, FAL_03). Estes documentos foram também armazenados do drive de nuvem junto com as gravações.

\subsection{Análise dos Dados}

A análise de dados foi realizada utilizando o método de análise de conteúdo, cruzando os dados obtidos pelas duas formas de coleta, procurando encontrar interligações entre as informações referentes as variáveis de classificação e as questões abertas.

A análise de conteúdo é um método que procura sistematizar e traduzir em dados mais objetivos informações que de origem qualitativa, explorando as características das diferentes formas de comunicação, por meio de procedimentos sistemáticos. O objetivo desta é a descrição do conteúdo da mensagem (Mendes \& Miskulin, 2017).

Primeiramente as entrevistas foram transcritas e foi realizada uma leitura exaustiva, procurando pontos em comum entre as respostas dadas pelos entrevistados e, assim, buscando padrões que se repetem entre as falas, o que permite a criação de categorias referentes as questões abertas da entrevista. Esta categorização é um passo importante para a análise de conteúdo, visto que permite relacionar temas que sejam próximo, contribuindo para uma visão mais ampla da temática.

Também foi feita uma análise do perfil dos pacientes utilizando a estatística descritiva. Esta procura avaliar e descrever as características de uma determinada amostra, resumindo diversos valores de uma mesma natureza (Mancuso et al., 2018). Utilizou-se para a análise, medidas de frequência, de tendência central e de dispersão, e, além disso, foram aplicados testes estatísticos para buscar alguma relação entre as variáveis dentro da amostra. Adotou-se o nível de confiança de $95 \%$ e erro padrão de 5\%. Os testes foram realizados com o auxílio do programa Sigma Plot®.

\section{Resultados e Discussão}

Com a extração inicial de dados, foram coletados 218 números telefônicos no total. A partir de então, começou-se a realizar as tentativas de ligação, a fim de completar a quantidade da amostra estimada em 139 participantes. Por conseguinte, foram realizadas tentativas de contato com todos os números, entretanto, não foi possível contactar 126 telefones. Nesse sentido, chegou-se ao número de 92 ligações bem sucedidas, das quais, 24 participantes foram excluídos por serem menores de 
idade, 19 foram excluídos por apresentarem erro de registro (o número não correspondia ao nome), 9 recusaram a participação, 5 não lembravam o motivo da falta, 4 afirmaram terem ido para a consulta, 3 foram consultados por outro profissional, 2 não realizaram a consulta pelo fato de o profissional ter faltado, 1 teve a consulta remarcada e 1 não realizou a entrevista completa. Com isso, obtiveram-se 24 entrevistados para a pesquisa.

Deve-se ressaltar os malefícios que os registros incorretos das marcações de consultas podem repercutir para a gestão da UBS. Isso porque impede com que os pacientes sejam contactados, o que poderia ser uma forma de diminuir o absenteísmo (Bueno et al., 2020). No caso do presente estudo, das 92 ligações bem sucedidas, 20,6\% ( $\mathrm{n}=19)$ foram excluídas por erros de registros.

\subsection{Perfil dos pacientes faltosos}

A amostra deste estudo possui um total de 24 participantes, dos quais, a maioria são do sexo feminino $(\mathrm{n}=18,75 \%)$. Esta preponderância do sexo feminino reflete principalmente o fato de que as mulheres são as principais usuárias das instâncias da Atenção Básica no Brasil (Ferreira et al., 2016). A idade média dos pacientes desta pesquisa foi de 33,9 anos. Médias de idades similares foram encontradas em outros estudos (Cavalcanti et al., 2013; Lenzi et al., 2019). Estas e outras características dos pacientes estão dispostas na Tabela 1 .

Tabela 1 - Perfil dos pacientes absente.

\begin{tabular}{|c|c|c|}
\hline \multicolumn{2}{|r|}{ Variável } & Paciente absente \\
\hline \multicolumn{2}{|r|}{ Idade (anos) } & $33,9 \pm 15,4$ \\
\hline \multicolumn{2}{|r|}{ Sexo (masculino) } & $6(25 \%)$ \\
\hline \multicolumn{2}{|r|}{ Escolaridade* } & $4(\mathrm{Q} 1=2,5 \mathrm{Q} 2=4)$ \\
\hline \multirow[t]{4}{*}{ Cor } & Negra & $6(25 \%)$ \\
\hline & Branca & $1(4,2 \%)$ \\
\hline & Parda & $17(70,8 \%)$ \\
\hline & $\mathrm{N}^{\circ}$ de filhos & $2(\mathrm{Q} 1=2 \mathrm{Q} 2=3,5)$ \\
\hline \multicolumn{2}{|r|}{ Cuida de dependente } & $2(8,3 \%)$ \\
\hline \multicolumn{2}{|r|}{ Possui veículo } & $3(12,5 \%)$ \\
\hline \multirow[t]{3}{*}{ Profissão } & Formal & $4(16,7 \%)$ \\
\hline & Informal & $4(16,7 \%)$ \\
\hline & Desempregado & $16(66,7 \%)$ \\
\hline \multicolumn{2}{|c|}{ Presença de doença grave ou crônica } & $5(20,8 \%)$ \\
\hline
\end{tabular}

Legenda: os dados estão expressos como: média \pm desvio padrão; frequência absoluta (frequência relativa); mediana (primeiro quartil terceiro quartil). *Dados de escolaridade estão de acordo com o seguinte score: 1 - nunca frequentou a escola; 2 - ensino fundamental incompleto; 3 ensino fundamental completo; 4- ensino médio completo; 5- ensino superior completo. Fonte: Autores.

Foi encontrado, neste estudo, uma mediana de pacientes faltosos com ensino médio completo. Neste sentido, esta amostra apresenta uma um grau de escolaridade elevado quando comparado com outros estudos que analisam o absenteísmo de pacientes odontológicos da rede pública (Almeida et al., 2010; Ferreira et al., 2016). Nestes estudos a maioria dos pacientes faltosos tinham ensino fundamental incompleto. Estas diferenças podem estar relacionadas ao serviço específico em que estes estudos focam.

Algumas variáveis analisadas nesta pesquisa apontam para um forte fator social presente nos pacientes da amostra. Pacientes não brancos representam 95,8\% $(\mathrm{n}=18)$ da nossa amostra, além de que 66,7\% ( $\mathrm{n}=16)$ de toda a amostra estava 
desempregado no momento da consulta e apenas $12,5 \%(\mathrm{n}=3)$ possuíam veículo. Estas determinantes sociais devem ser consideradas para que o acesso aos serviços de saúde sejam melhor aproveitados pela população (Moura Louzada Farias et al., 2020).

Foram feitos testes estatísticos para encontrar relações entre as variáveis estudadas dentro da amostra. Desta forma, o teste de correlação de Pearson apontou uma correlação positiva entre a idade dos pacientes e a quantidade de filhos, com um coeficiente de correlação de 0,553 ( $\mathrm{p}=0,0114$ ) (Figura 2). Outrossim, o teste de Kruskal-Wallis apontou uma diferença de idade mediana entre os pacientes portadores ou não de doença grave ou crônica $(p=0,012)$, neste caso, o teste de pareamento de Dunn apontou que esta variação estava entre pacientes saudáveis (mediana de 26 anos) e pacientes com diabetes e hipertensão simultaneamente (mediana de 66 anos) (Figura 3).

Figura 2 - Gráfico da mediana, $1^{\circ}$ e $3^{\circ}$ quatis da idade em relação a presença de doença grave ou crônica.

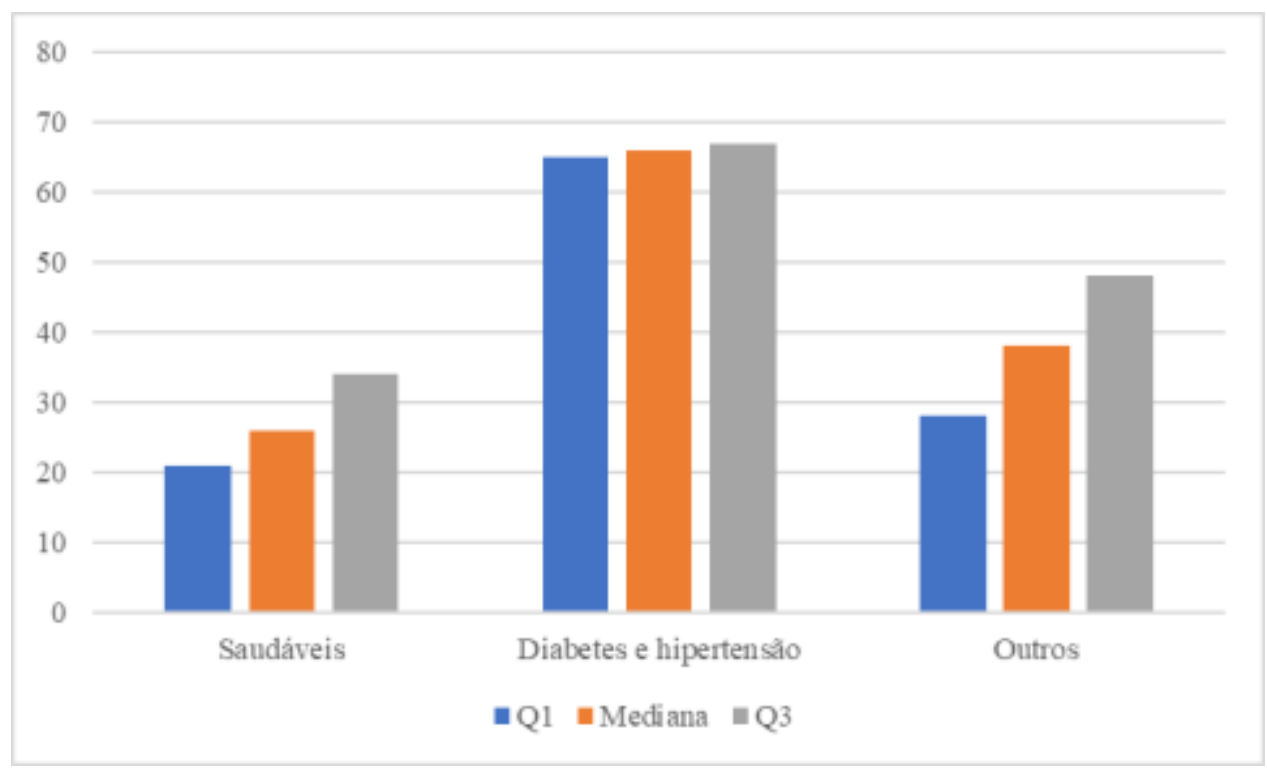

Fonte: Autores.

Figura 3 - Gráfico de dispersão (idade vs nº de filhos).

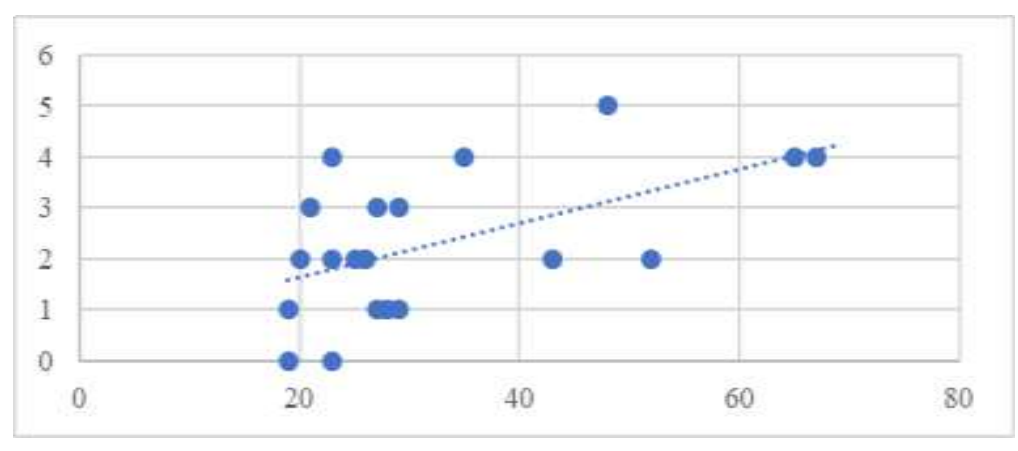

Fonte: Autores.

Estas relações apontadas pelos testes estatísticos podem significar que a idade dos pacientes esteja atrelada a outras variáveis, o que pode significar que ela seja uma das mais importantes características que devem ser analisadas na busca de criação de ações afirmativas que visem combater o absenteísmo. A relação da idade com o número de filhos e as condições pesquisadas já era esperada nesta população, visto que a ESF possuí programas específicos para as três populações, além de serem indicativos do público que frequenta a UBS (Silva et al., 2019). 
Foram aplicados testes nas outras combinações de variáveis, contudo todos os resultados não apresentavam relevância estatística $(\mathrm{p}>0,05)$.

\subsection{Motivações do absenteísmo dos pacientes}

\subsubsection{A gestão da unidade}

O absenteísmo de pacientes relacionado a gestão das unidades é bem documentado na literatura (Boos et al., 2016; Costa et al., 2018; Ferreira et al., 2016; Silveira et al., 2019). Outrossim, um dos entrevistados aponta o não funcionamento da Regulação como fator preponderante na sua falta à consulta, pois não conseguiu realizar os exames que necessitava a tempo da consulta de retorno. Essas limitações de funcionamento impedem com que a UBS atenda de forma completa as necessidades da população assistida (Silva et al., 2019).

"A regularização não estava funcionando pra fazer os exames" (FAL_12).

Outro ponto que deve ser encarado como um fator causador de absenteísmo são as barreiras comunicativas entre os pacientes e os trabalhadores das unidades de saúde. Ruídos na comunicação podem fazer com que o entendimento do paciente seja prejudicado e o mesmo deixe de ir as consultas por não ter compreendido que deveria fazê-lo (Abelsson et al., 2020).

"Não sabia que tinha consulta marcada pra mim" (FAL_20).

"Não me disseram que havia consulta marcada em meu nome" (FAL_17).

Um dos motivos apontados pelos pacientes foi o tempo de espera elevado. Além disso, muitas vezes, esta demora pode fazer com que os usuários procurem outros locais para realizar os procedimentos necessários, o que pode culminar em um aumento de gastos, pois podem procurar serviços particulares para que o processo seja agilizado (Costa et al., 2018). Este absenteísmo por troca de instituição está relacionado, nesta pesquisa, com a visão do paciente sobre a gravidade do próprio estado e, consequentemente, a procura por um atendimento melhor ou mais rápido, na sua visão.

"Minha situação era de emergência, e como a consulta iria demorar muito, consegui realizar a consulta em outra unidade em um prazo menor" (FAL_18).

"Meu caso era de emergência, não dava pra esperar até a consulta, já fui direto fazer a cirurgia" (FAL_24).

"É porque a data prevista do meu parto seria o dia primeiro de janeiro (a consulta estava marcada para o dia posterior) [...] no dia que seria a consulta, eu fui pro HMI" (FAL_1).

\subsubsection{Caracterização do usuário}

\subsubsection{Horário e data}

A disponibilidade do paciente a comparecer na consulta é um fator que se apresentou preponderante em nossa pesquisa e também é relatado na literatura (Costa et al., 2018). Ainda neste motivo, destacou-se que alguns pacientes não se encontravam na cidade ou estavam comprometidos com outras atividades. Nesses casos, seria interessante fazer contato com os pacientes um dia antes para confirmar sua presença, e este contato poderia ser feito por mensageiros online, por exemplo (Bueno et al., 2020).

"Foi uma viagem que eu fiz, e aí não deu de eu vir na data" (FAL_2).

"Estava viajando na data da consulta" (FAL_16).

O funcionamento das UBS em horário similar ao horário de trabalho da maioria dos usuários provavelmente explique o fato de ser este o maior motivo de faltas às consultas (Gonçalves et al., 2015).

"Tinha umas coisas pra mim resolver, acabei não indo" (FAL_5).

"É porque eu tinha outro compromisso particular na mesma data da consulta" (FAL_14).

"Eu fui para o pré-vestibular fazer uma prova" (FAL_15). 
"Tive que ir trabalhar" (FAL_21).

Consultas marcadas simultâneas têm se mostrado um problema gerador de absenteísmo (Cavalcanti et al., 2013). Isto ocorre porque o paciente precisa, então, decidir qual serviço deve priorizar, deixando de ir ao outro.

"Porque eu estava com outra consulta lá no CRISMU" (FAL_11).

O esquecimento do paciente foi apontado como a motivação da maioria das faltas no estudo de Ferreira et al. (2016). Entretanto, nesta pesquisa, esse fato não se mostrou tão preponderante, apesar de ser apontado por dois dos entrevistados. O paciente precisa ser devidamente informado sobre o dia da consulta agendada e o horário, qualquer falha nessa comunicação ao usuário sobre o correto agendamento gera um absenteísmo "involuntário" do mesmo.

"Não lembrei que havia consulta marcada" (FAL_19).

"Eu confundi as datas" (FAL_23).

\subsubsection{Deslocamento e localização da unidade}

Deve-se salientar que os principais usuários das UBS's são pessoas de renda mais baixa (Silva et al., 2019). Deste modo, é importante que o serviço seja acessível para esta população, contudo, a disponibilidade de um transporte para se dirigir a unidade torna-se um fator gerador de absenteísmo.

"Por falta do dinheiro do transporte" (FAL_9).

De acordo com (Moura Louzada Farias et al., 2020), as barreiras geográficas e dificuldades de transporte, devido à localização desses serviços, no que se refere aos locais de residência e de trabalho dos entrevistados, também foram identificados como determinantes relevantes, na perspectiva particular dos usuários, que podem impactar diretamente no absenteísmo às consultas e exames previamente agendados.

“É porque eu resolvi fazer o meu pré-natal aqui, no posto mais próximo da minha casa” (FAL_3).

"No momento eu não tô em Marabá, tô em nova Ipixuna, na casa da minha mãe, vim passar o resguardo pra cá" (FAL_6).

"Mas foi por causa do transporte, porque meu marido tava viajando. [...] Aí não tinha ninguém pra me acompanhar também" (FAL_7).

\subsubsection{Outros fatores}

\subsubsection{Fatores ambientais}

Dentre os fatores que chamaram a atenção por serem pouco discutidos nas literaturas de base existentes, estão os fatores relacionados ao clima, dos quais $8 \%(\mathrm{n}=2)$ dos entrevistados relataram não ir à consulta por conta da chuva. A partir disso, denota-se que os aspectos climáticos podem se tornar barreiras na adesão aos serviços de saúde. Além disso, esses fatores podem estar associados às características socioeconômicas dos pacientes e, também, a responsabilidades secundárias de cuidador de algum dependente, por parte dos pacientes. Deste modo, os absentes, tendo como base o fator chuva, relataram não possuírem transporte próprio, situação financeira favorável para arcar com serviços de transporte particular e cuidadores, que lhes garantissem condições adequadas de se fazerem presentes nas consultas.

"Era porque tava chovendo muito." (FAL_8)

"Nesse dia tava chovendo muito, aí eu não queria deixar o meu bebê." (FAL_10)

\subsubsection{Relação médico-paciente}

A construção de um laço entre o paciente e profissional é muito importante, e deve ser mantida durante o atendimento. Uma mudança de profissional pode interromper o tratamento do paciente, podendo causar problemas na esfera de 
responsabilidade civil, levando em consideração a forma com que o médico aborda o paciente. Para Rodrigues et al. (2006), ouvir e se comunicar com o paciente e/ou tutor deve passar por todas as etapas da produção de saúde, e são fatores para o sucesso da prática médica. Um usuário relata não ter comparecido por não se sentir à vontade durante a consulta. É possível identificar que não foi estabelecida uma afinidade e segurança com o profissional. Neste caso, segundo Costa, Duarte e Vaghetti (2018), a integralidade da assistência favorece a continuidade do atendimento ao indivíduo.

"Não gostei de como o médico me tratou, ele era muito arrogante" (FAL_22).

\subsubsection{Desconhecimento do paciente sobre os procedimentos e saúde do paciente}

Os dois últimos fatores descrevem aspectos intrínsecos da população humana e levam em consideração acontecimentos de caráter multifatorial. Dentre eles, o fato de uma paciente não possuir condições adequadas de saúde para comparecer a consulta, induz a uma reflexão sobre qual seria o objetivo principal dos pacientes ao procurarem os serviços de saúde pública, discussão essa, que perpassa através das possibilidades de se buscar atendimento em caráter preventivo/profilático ou somente diante de estados de moléstia (Izecksohn \& Ferreira, 2014). Por conseguinte, uma participante relata não ter comparecido a consulta pelo desconhecimento de orientações sobre qual a característica fisiológica, relacionada ao ciclo menstrual, seria necessária apresentar, na data da consulta, para se fazer presente na avaliação ginecológica.

"Minha cirurgia de retirada do útero foi marcada próxima a data da consulta, porém nesse dia eu estava me sentindo mal" (FAL_13).

"Não sabia como é que funcionava. No dia do exame, eu achei de ficar menstruada, bem no segundo dia, bem no dia que o fluxo é maior. Então assim, eu coloquei na minha cabeça ‘não, não vou fazer”' (FAL_4).

\subsection{Consequências do absenteísmo}

Além dos motivos que ocasionaram as faltas dos pacientes às consultas agendadas, prosseguiu-se questionando aos entrevistados, de acordo com sua opinião, quais seriam as principais consequências do seu absenteísmo. Nesse sentido, as respostas foram agrupadas em quatro categorias de acordo com a diversidade dos relatos.

Dentre as respostas obtidas, a maioria dos usuários, cerca de $33 \%$ relataram terem consciência do aspecto negativo de ser um paciente absente para a dinâmica da UBS e que a consequência mais expressiva do seu não comparecimento fica a cargo de outros pacientes que poderiam ter utilizado a vaga na consulta, mas não puderam por já ter sido preenchida. Nesse quesito, torna-se bastante plausível uma reflexão à cerca da necessidade de se construir um diálogo permanente entre paciente e UBS a fim de construir mecanismos que ofereçam oportunidades de comunicar antecipadamente, sempre que possível, o não comparecimento e, com isso, a provável realocação da vaga para outro usuário do serviço.

Cerca de $25 \%$ dos entrevistados acreditam que a sua falta à consulta tem como principal prejudicado o próprio paciente. Nessa perspectiva, foi destacado como caráter negativo fatores que ilustram o prejuízo para o paciente por não ter ido à consulta: o longo período de espera para a realização de outra consulta; a demora na realização de exames pelo laboratório público da UBS; a interrupção de tratamento de saúde, por conta da não renovação de receita, para aqueles pacientes que fazem uso regular de medicação por conta de doenças crônicas como, por exemplo, na hipertensão arterial e na diabetes.

Outrossim, uma categoria que possui significativa relevância a ser discutida é o fato de $25 \%$ dos pacientes absentes relatarem não terem conhecimento sobre qual seria a consequência negativa da sua falta à consulta. Diante disso, é indubitável levar em consideração não somente o desconhecimento das possíveis repercussões, mas também, o aspecto de irrelevância que o absenteísmo representa para o paciente no que concerne as suas preocupações cotidianas (Izecksohn \& Ferreira, 2014). 
Em relação a quarta categoria, aproximadamente $16 \%$ dos entrevistados expressaram não reconhecerem consequências negativas diante da sua falta, afinal, de acordo com os próprios, as equipes responsáveis pelos procedimentos de marcação das consultas atuam realocando outros pacientes nas vagas ociosas. Essa é uma informação que chama bastante atenção pelo fato de os pacientes procurarem a UBS, mesmo não possuindo consulta marcada na data, por levarem em consideração o conhecimento prévio de que a taxa de absentes diária é expressiva, e que os funcionários realizam adequações nas marcações de acordo com a demanda (Brasil, 2017).

\section{Conclusão}

O perfil dos pacientes absentes encontrados nessa pesquisa apresentou características de vulnerabilidade social. A maioria dos pacientes possuíam indicativos de renda baixa (não tinham posse de veículo), além disso, grande parte deles estavam desempregados no momento da entrevista. Isto demonstra que o absenteísmo está interligado a fatores sociais, logo é imperativo que os serviços oferecidos levem em consideração estas fragilidades.

Outrossim, problemas na gestão e efetivação de serviços da própria UBS induziram o absenteísmo. Este fato demonstra que o sistema de controle de consultas da UBS precisaria de revisão para que se adeque melhor as necessidades da população assistida.

Ademais, a percepção dos pacientes em relação aos prejuízos causados pela sua falta se mostra diversificada. Dentro dessa variedade de percepções destacam-se o entendimento do dano causado a outros usuários do sistema de consulta, a negligência com as repercussões do absenteísmo, o paciente como principal sujeito prejudicado e o desconhecimento das consequências. É importante a criação de mecanismos que solidifiquem o diálogo entre unidade e usuários, a fim de estabelecer uma relação mais transparente e compactuada entres os sujeitos e seus papeis na dinâmica da saúde pública.

Este trabalho apresentou limitações em relação ao tamanho da amostra, o que torna conclusões acerca do perfil dos pacientes pouco expressivas. Outrossim, os mecanismos de análise estatística, por esse motivo, não apresentaram forte poder estatístico. Estas limitações estão relacionadas com a falta de um mecanismo eficiente de registro do contato dos pacientes, muitos números foram descartados por não terem sido registrados de forma correta, logo, torna-se necessário a criação de um modo sistematizado de registro dos telefones dos pacientes na presente UBS.

Espera-se que, no futuro, trabalhos que abranjam maior e mais diversamente localizada amostra, a fim de compor mais dados primários sobre o assunto, outrossim, trabalhos que verifiquem diretamente a influencia entre um sistema efetivo de registro e contato de pacientes e o absenteísmo dessa população ajudaria a esclarecer mais esta relação.

\section{Referências}

Abelsson, T., Morténius, H., Bergman, S., \& Karlsson, A.-K. (2020). Quality and availability of information in primary healthcare: the patient perspective. Scandinavian Journal of Primary Health Care, 38(1), 33-41. https://doi.org/10.1080/02813432.2020.1718311

Almeida, G. L. de, Garcia, L. da F. R., Almeida, T. L. de, Bittar, T. oliveira, \& Pereira, A. C. (2010). Estudo do perfil sócio-econômico dos pacientes e os motivos que os levaram a faltar em consultas odontológicas na estratégia de saúde da família em uma distrital de Ribeirão Preto/SP. Brazilian Dental Science, 12(1), 77-86. https://doi.org/10.14295/bds.2009.v12i1.256

Batista, E. C., Matos, L. A. L., \& Nascimento, A. B. (2017). A entrevista como técnica de investigação na pesquisa qualitativa. Revista Interdisciplinar Científica Aplicada, 11(3), 23-38.

Boos, E. M., Bittner, M. J., \& Kramer, M. R. (2016). A Profile of Patients Who Fail to Keep Appointments in a Veteran Affairs Primary Care Clinic. WMJ, $115(4), 185-190$.

Brasil. (2017). Portaria n ${ }^{\circ}$ 2.436, de 21 de setembro de 2017. Aprova a Política Nacional de Atenção Básica, estabelecendo a revisão de diretrizes para a organização da Atenção Básica, no âmbito do Sistema Único de Saúde (SUS). Diário Oficial da União, 183(1).

Bueno, N. da S., Rossoni, A. M. de O., Lizzi, E. A. da S., Tahan, T. T., Hirose, T. E., \& Chong Neto, H. J. (2020). How Can New Technologies Help Reduce Absenteeism In Pediatric Consultation? Revista paulista de pediatria: orgao oficial da Sociedade de Pediatria de Sao Paulo, 38 , e2018313. https://doi.org/10.1590/1984-0462/2020/38/2018313 
Cavalcanti, R., Cavalcanti, R. P., Cavalcanti, J. C. M., Serrano, R. M. S. M., \& Santana, P. R. (2013). Absenteísmo de consultas especializadas nos sistema de saúde público: relação entre causas e o processo de trabalho de equipes de saúde da família, João Pessoa - PB, Brasil. Tempus Actas de Saúde Coletiva, 7(2), Pág. 63-84. https://doi.org/10.18569/tempus.v7i2.1344

Cechinel, A., Pereira Fontana, S. A., Pazeto Della Giustina, K., Serafim Pereira, A., \& Salvador do Prado, S. (2016). Estudo/Análise Documental: Uma Revisão Teórica E Metodológica. Criar Educação, 5(1). https://doi.org/10.18616/ce.v5i1.2446

Costa, C. F. S. da. (2018). Absenteísmo Em Consultas Especializadas Referenciadas Por Unidade Básica Saúde Da Família: Estudo De Caso. Saúde (Santa Maria), 44(1). https://doi.org/10.5902/2236583420922

Costa, C. F. S. da, Duarte, P. M., \& Vaghetti, H. H. (2018). Absenteísmo em Consultas Especializadas Referenciadas por Unidade Básica Saúde da Família: Estudo de Caso. Saúde (Santa Maria), 44(1). https://doi.org/10.5902/2236583420922

Ferreira, M. B., Lopes, A. C., Lion, M. T., Lima, D. C. de, Nogueira, D. A., \& Pereira, A. A. (2016). Absenteísmo Em Consultas Odontológicas Programáticas Na Estratégia Saúde Da Família. revista da universidade vale do rio verde, 14(1), 411-419. https://doi.org/10.5892/ruvrd.v14i1.2601

Gonçalves, C. Â., De Lima Vazquez, F., Ambrosano, G. M. B., Mialhe, F. L., Pereira, A. C., Sarracini, K. L. M., Guerra, L. M., \& Cortellazzi, K. L. (2015). Estratégias para o enfrentamento do absenteísmo em consultas odontológicas nas Unidades de Saúde da Família de um município de grande porte: Uma pesquisa-ação. Ciencia e Saude Coletiva, 20(2), 449-460. https://doi.org/10.1590/1413-81232015202.00702014

Hallingberg, B., Turley, R., Segrott, J., Wight, D., Craig, P., Moore, L., Murphy, S., Robling, M., Simpson, S. A., \& Moore, G. (2018). Exploratory studies to decide whether and how to proceed with full-scale evaluations of public health interventions: A systematic review of guidance. In Pilot and Feasibility Studies (Vol. 4, Número 1, p. 104). BioMed Central Ltd. https://doi.org/10.1186/s40814-018-0290-8

IBGE. (2010). Censo demográfico: população no último censo de Marabá.

Izecksohn, M. M. V., \& Ferreira, J. T. (2014). Falta às consultas médicas agendadas: percepções dos usuários acompanhados pela Estratégia de Saúde da Família, Manguinhos, Rio de Janeiro. Revista Brasileira de Medicina de Família e Comunidade, 9(32), 235-241. https://doi.org/10.5712/rbmfc9(32)960

Kim, H., Sefcik, J. S., \& Bradway, C. (2017). Characteristics of Qualitative Descriptive Studies: A Systematic Review. Research in Nursing and Health, 40(1), 23-42. https://doi.org/10.1002/nur.21768

Lenzi, H., Ben, Â. J., \& Stein, A. T. (2019). Development and validation of a patient no-show predictive model at a primary care setting in Southern Brazil. PLoS ONE, 14(4). https://doi.org/10.1371/journal.pone.0214869

Macinko, J., \& Mendonça, C. S. (2018). Estratégia Saúde da Família, um forte modelo de Atenção Primária à Saúde que traz resultados. Saúde em Debate, 42(spe1), 18-37. https://doi.org/10.1590/0103-11042018s 102

Mancuso, A. C. B., Castro, S. M. de J., Guimarães, L. S. P., Leotti, V. B., Hirakata, V. N., \& Camey, S. A. (2018). Estatística descritiva: perguntas que você sempre quis fazer, mas nunca teve coragem. Clinical \& Biomedical Research, 38(4), 414-418. https://doi.org/10.4322/2357-9730.89242

Mendes, R. M., \& Miskulin, R. G. S. (2017). A análise de conteúdo como uma metodologia. Cadernos de Pesquisa, 47(165), 1044-1066. https://doi.org/10.1590/198053143988

Moura Louzada Farias, C., Moraes, L., Esposti, C. D. D., Santos Neto, E. T., Profissional, M., Administração, E. M., Ferreira, M. B., Lopes, A. C., Lion, M. T., Lima, D. C. de, Nogueira, D. A., Pereira, A. A., \& Costa, C. F. S. da. (2020). Absenteísmo de usuários: barreiras e determinantes no acesso aos serviços de saúde. Revista Da Universidade Vale Do Rio Verde, 14(1), 411-419. https://doi.org/10.5712/rbmfc15(42)2239

Pereira, A. R. (2015). A cidade invisível de Marabá. In E. Cavalcanti \& G. Cabral (Orgs.), A história e suas práticas de escrita (p. 51-78). Editora UFPE.

Pocinho, M., \& Figueredo, J. P. (2004). Estatística e Bioestatística. Madeira.

Rodrigues, C. K., Shintcovsk, R. L., Tanaka, O., França, B. H. S., \& Hebling, E. (2006). Responsabilidade civil do ortodontista. Revista Dental Press de Ortodontia e Ortopedia Facial, 11(2), 120-127. https://doi.org/10.1590/s1415-54192006000200015

Silva, M. A., Teixeira, S. S., \& Libarino, C. (2019). As Limitações da Gestão Pública no Atendimento às Necessidades dos Usuários de um Posto de Saúde em Vitória da Conquista - BA. Revista Innovare, 28, 193-215.

Silveira, G. S. da, Ferreira, P. R. de, Silveira, D. S. da, \& Siqueira, F. C. V. (2019). Prevalência de absenteísmo em consultas médicas em unidade básica de saúde do sul do Brasil. Revista Brasileira de Medicina de Família e Comunidade, 13(40), 1-7. https://doi.org/10.5712/rbmfc13(40)1836

Troncoso-Pantoja, C., \& Amaya-Placencia, A. (2017). Interview: A practical guide for qualitative data collection in health research. Revista Facultad de Medicina, 65(2), 329-332. https://doi.org/10.15446/revfacmed.v65n2.60235

UBS.MED.BR. (2018). Centro de Saúde Laranjeiras - Marabá - PA - Centro de Saúde, Unidade Básica. 